\title{
Expression and mechanism of microRNA-181A on incidence and survival in late liver metastases of colorectal cancer
}

\author{
ZENGJUN LI, HAIPENG WANG, ZHONGFA XU, YANLAI SUN and JIANJUN HAN \\ Department of General Surgery, Shandong Cancer Hospital and Institute, \\ Shandong Academy of Medical Science, Jinan, Shandong 250117, P.R. China
}

Received August 14, 2015; Accepted October 1, 2015

DOI: $10.3892 /$ or.2016.4546

\begin{abstract}
The emerging role of microRNA-181A (miR-181A) in CRC patients with late liver metastases was studied. In the present study we investigated the association between expression and mechanism of miRNA-181A, liver metastasis and prognosis of colorectal cancer (CRC). The expression of miR181A and PTEN in CRC patients with late liver metastases was higher than that of the normal (control) group, whereas the expression of miR-181A and PTEN was lower in all pathological groups (TNM I-TNM IV). Overall survival (OS) of lower expression miR-181A CRC patients with late liver metastases was higher than that of higher expression miR-181A CRC patients with late liver metastases. The expression of miR-181A and PTEN in the colon cell line NCM460 was lower than that of the colon cancer SW620 cell line. Upregulation of miR-181A promoted cell viability and inhibited apoptosis of SW620 cells, suppressed PTEN expression and activated phosphorylation of AKT (p-AKT) in SW620 cells. Additionally, downregulation of miR-181A inhibited cell viability of SW620 cells through promotion of PTEN and inhibition of p-AKT. Together, our results indicate that miR-181A expression is associated with CRC patients with late liver metastases through PTEN/AKT signaling.
\end{abstract}

\section{Introduction}

Colorectal cancer (CRC) is one of the most common malignant types of cancer with the third highest mortality rate (1). The diagnosis and treatment of CRC can be quite difficult as the molecular mechanisms are as yet not fully elucidated. To date, the most effective therapy is surgery combined with chemotherapy and the 5-year survival rate of a local tumor is $\sim 93 \%(2,3)$. However, nearly half of patients have metastasis when they are first diagnosed and more than a third relapse

Correspondence to: Dr Zengjun Li, Department of General Surgery, Shandong Cancer Hospital and Institute, Shandong Academy of Medical Science, Jinan, Shandong 250117, P.R. China

E-mail: lizengjunjun250117@163.com

Key words: miR-181A, colorectal cancer, late liver metastases, PTEN, AKT after surgery. Even with advanced surgical techniques and new molecular therapies, death rates for CRC patients are still high, and this is largely due to recurrence of cancer due to metastasis (4).

The molecular mechanism that drive CRC in terms of progression as well as invasion and metastasis have not been fully elucidated. It is generally thought that occurrence and progression of CRC is a multi-factor, multi-phase, multi-step and multi-gene process $(5,6)$. Most of the mutations that drive CRC occur in oncogenes driving activation or in tumor suppressor genes to causing inactivation (7). Numerous different studies have shown that the occurrence and progression of CRC, as well as invasion and metastasis, involve many related genes. For example, mutations in c-myc, Ras, Her-2 and the cancer suppressor gene p53, which is deleted in colorectal carcinoma, can drive occurrence. Other genes involved in CRC progression include PTEN (phosphatase and tensin homologue, deleted on chromosome 10), a metastasis suppressor gene nm23 and its encoding genes, such as CD44, MMPs, MUC and dMMR. The loss of DNA mismatch repair mechanisms also drives progression as well as mutations in KRAS and BRAF $(8,9)$. Therefore, the molecular mechanisms are complicated.

The occurrence and progression of a given cancer is a multi-factor and multi-step process, in which abnormal gene expression plays a vital role (10). However, with continued research into pathogenic mechanisms of CRC, molecular markers that play an essential role in the early diagnosis and drive clinical progression, have become more apparent and have led to the concept of individual-based treatment being a realistic goal (9). In addition, this increased knowledge of biomarkers will undoubtedly assist in earlier diagnosis and more accurate monitoring of disease progression. To date, nearly 1500 miRNA sequences have been found. Studies of 186 miRNA locus, have suggested that miRNA may be essential for the occurrence and development of many different types of cancer (11). In the present study, we correlated the expression of miR-181a with incidence and survival, in patients with late liver metastases of CRC.

\section{Materials and methods}

Tissue specimens and cell cultures. CRC tissue and non-cancer liver tissue were obtained from patients with colorectal cancer. 
All tissues were stored in liquid nitrogen. Written informed consent was signed by all participating patients. This study was approved and permitted by the Ethics Committee of Shandong Cancer Hospital and Institute. All clinical and histopathological indexes were rendered anonymous. The human colon NCM460 cells and human colon cancer cells SW620 were cultured with Dulbecco's modified Eagle's medium (DMEM) and 10\% fetal bovine serum (FBS), $100 \mathrm{U} /$ $\mathrm{ml}$ penicillin and $100 \mathrm{mg} / \mathrm{ml}$ streptomycin (Invitrogen, Carlsbad, CA, USA).

Survival analysis. Overall survival (OS) was evaluated in 72 CRC patients with late liver metastases and 69 normal control patients from Shandong Cancer Hospital and Institute. The survival curves were analyzed using the Kaplan-Meier method and statistically compared using a log-rank test.

$R N A$ extraction and real-time quantitative RT-PCR. Total RNA was extracted from tissue samples and NCM460 and SW620 cells using TRIzol reagent (Invitrogen). Total RNA $(2 \mu \mathrm{g})$ was used for reverse transcriptase cDNA Moloney murine leukemia virus reverse transcriptase (Invitrogen) according to the manufacturer's protocol. Real-time PCR was performed using SsoFast ${ }^{\mathrm{TM}}$ EvaGreen Supermix (Bio-Rad Laboratories) and the SYBR-Green PCR Master Mix (Toyobo Co., Ltd., Osaka, Japan). PCR cycling conditions were: $95^{\circ} \mathrm{C}$ for $45 \mathrm{sec}, 40$ cycles of $95^{\circ} \mathrm{C}$ for $45 \mathrm{sec}, 60^{\circ} \mathrm{C}$ for $30 \mathrm{sec}$ and $72^{\circ} \mathrm{C}$ for $30 \mathrm{sec} .2^{-\Delta \mathrm{Ct}}$ where $\Delta \mathrm{Ct}=\mathrm{Ct}$ (Target) $-\mathrm{Ct}$ (Reference).

Cell viability assay. Cell viability of NCM460 and SW620 cells was determined using MTT assay (Sigma-Aldrich, St. Louis, MO, USA). Briefly, NCM460 and SW620 cells were seeded in 96-well plates $\left(1-2 \times 10^{3}\right.$ cells/well $)$ and cultured at $37^{\circ} \mathrm{C}$ in a humidified atmosphere of $5 \% \mathrm{CO}_{2}$ overnight. For the MTT assay, $20 \mu \mathrm{l}$ of MTT was added into each well and incubated at $37^{\circ} \mathrm{C}$ for an additional $4 \mathrm{~h}$. The absorbance was detected at $490 \mathrm{~nm}$ using a plate microreader (Perkin-Elmer, San Diego, CA, USA).

Apoptosis. Cell apoptosis was determined using Annexin VFITC/propidium iodide assay (Medical and Biological Laboratories Co., Woburn, MA, USA). Briefly, NCM460 and SW620 cells were seeded in 96-well plates $\left(1-2 \times 10^{6}\right.$ cells/well $)$ and cultured at $37^{\circ} \mathrm{C}$ in a humidified atmosphere of $5 \% \mathrm{CO}_{2}$ overnight. Annexin V-FITC (10 $\mu \mathrm{l})$ was added to cells and incubated for $30 \mathrm{~min}$ in the dark. Propidium iodide was added into stained cells and immediately analyzed by flow cytometry (Beckman Coulter, Brea, CA, USA).

Western blotting. Total protein lysates were extracted from tissue and cell samples using lysed on ice in a buffer (Beyotime Institute of Biotechnology, Haimen, China), and then quantitated using BCA protein assay kit (Pierce, Rockford, IL, USA). Equal protein was resolved on $12 \% \mathrm{w} / \mathrm{v}$ SDS-PAGE gel and transferred onto polyvinylidene difluoride (PVDF) membrane (GE Healthcare Life Sciences, Piscataway, NJ, USA). The membrane was blocked with TBST containing 5\% skim milk powder followed by incubation with rabbit rabbit antiphosphorylated AKT (1:2,000; Santa Cruz Biotechnology, Santa Cruz, CA, USA) and rabbit anti-AKT (1:2;000; Santa
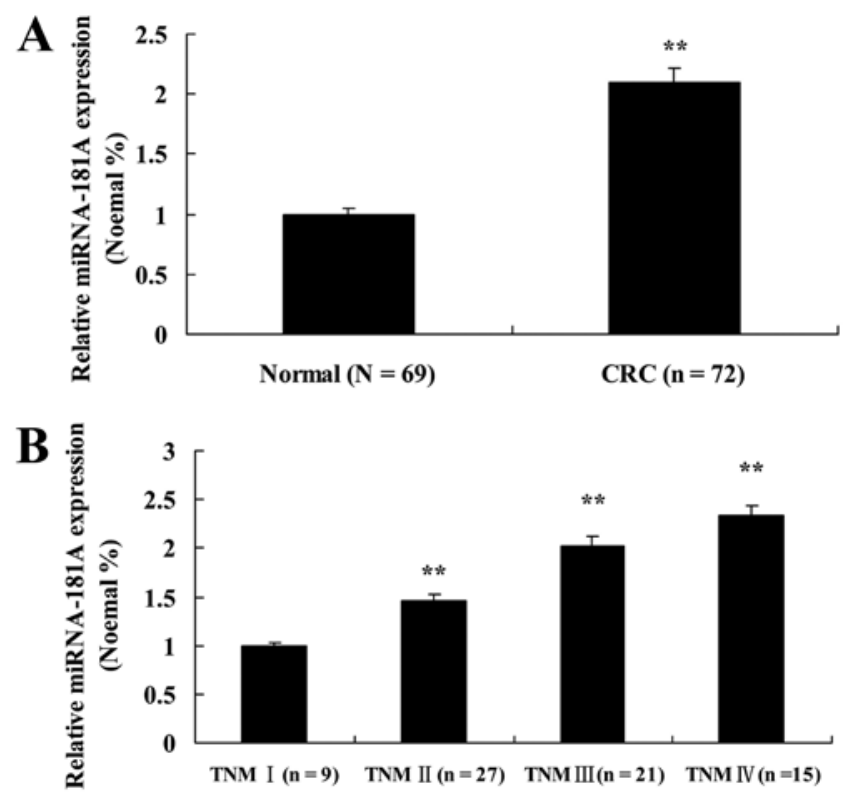

Figure 1. The expression of miR-181A in CRC patients with late liver metastases. The expression of miR-181A in CRC patients with (A) late liver metastases and (B) pathological somatotype. ${ }^{* *} \mathrm{P}<0.01$ compared with normal group.

Cruz Biotechnology) at $4^{\circ} \mathrm{C}$ overnight. The membrane was incubated with secondary horseradish peroxidase-conjugated goat anti-rabbit or anti-mouse antibody (Beyotime Institute of Biotechnology).

Establishment of stable cell lines. NCM460 and SW620 cells were seeded onto 6-well plates at $\sim 70-80 \%$ confluence. NCM460 and SW620 cells were transfected with miR-181A, anti-miR-181A or miR-negative control (NC) using Lipofectamine 2000 (Invitrogen) according to the manufacturer's instructions. The expression of miR-181A was confirmed by real-time quantitative RT-PCR, as described above.

Statistical analysis. The experimental data are presented as the mean \pm standard deviation (SD) and performed with the SPSS 17.0 software using the Kaplan-Meier method and statistically compared using a log-rank test. Differences were considered statistically significant at $\mathrm{P}$-values $<0.05$.

\section{Results}

The expression of miR-181A in CRC patients with late liver metastases. To investigate the expression of miR-181A in CRC patients with late liver metastases, we used a microarray and examined expression of miR-181A in 72 CRC patients and 69 normal (control) patients, from Shandong Cancer Hospital and Institute. The expression of miR-181A in CRC patients with late liver metastases was higher than that of the control group (Fig. 1A), whereas the expression of miR-181A was lower in all the pathological groups (TNM I-TNM IV) (Fig. 1B).

The expression of miR-181A is associated with survival in late liver metastases. To examine whether the expression of miR-181A is associated with survival in late liver metastases, 


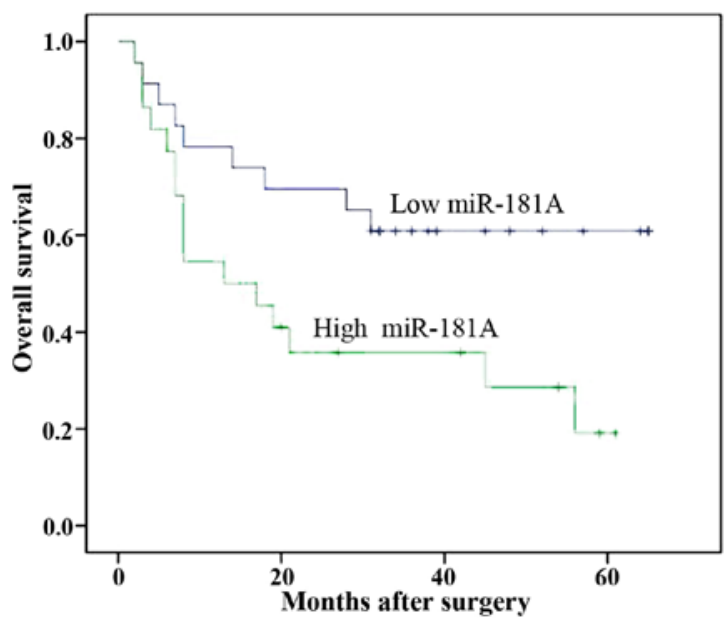

Figure 2. The expression of miR-181A is associated with survival in late liver metastases.
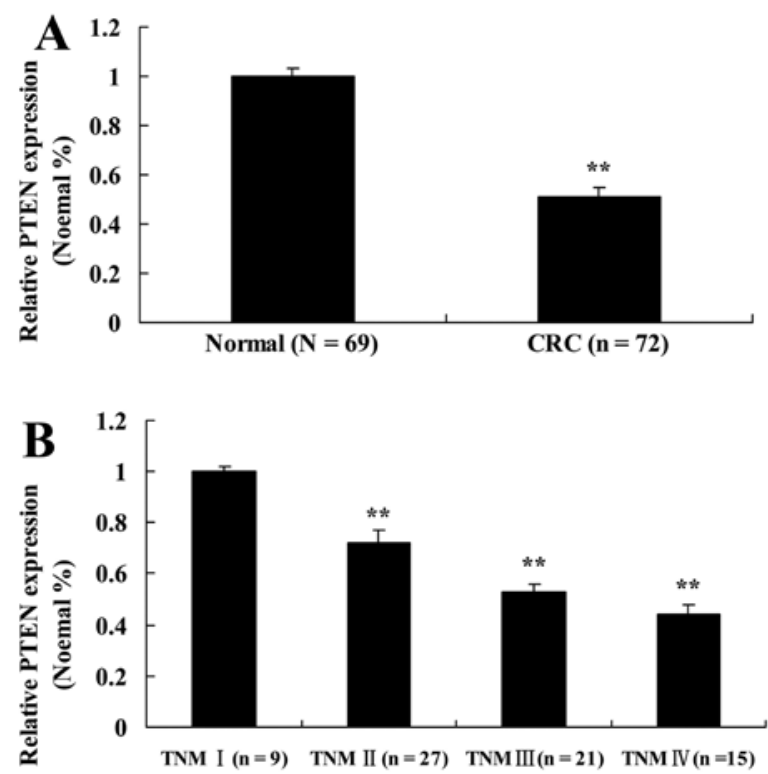

Figure 3. The PTEN in CRC patients with late liver metastases. The PTEN in CRC patients with (A) late liver metastases and (B) pathological somatotype. ${ }^{* *} \mathrm{P}<0.01$ compared with normal group.

we compared the overall survival (OS) between CRC patients (with late liver metastases) with lower miR-181A with CRC patients with higher miR-181A (Fig. 2).

The PTEN in CRC patients with late liver metastases. To further elucidate the mechanism of miR-181A on CRC patients with late liver metastases, we examined the expression of PTEN. Quantitative RT-PCR analyses were carried out in 72 CRC patients with late liver metastases and 69 normal patients. qRT-PCR analysis revealed a significant decrease in PTEN expression in CRC patients with late liver metastases compared to normal patients (Fig. 3A). In addition, the expression of PTEN in TNM I CRC patients was markedly enhanced compared to other pathological groups (Fig. 3B). This result suggests that there is a specific association between miR-181A, PTEN and CRC metastases.

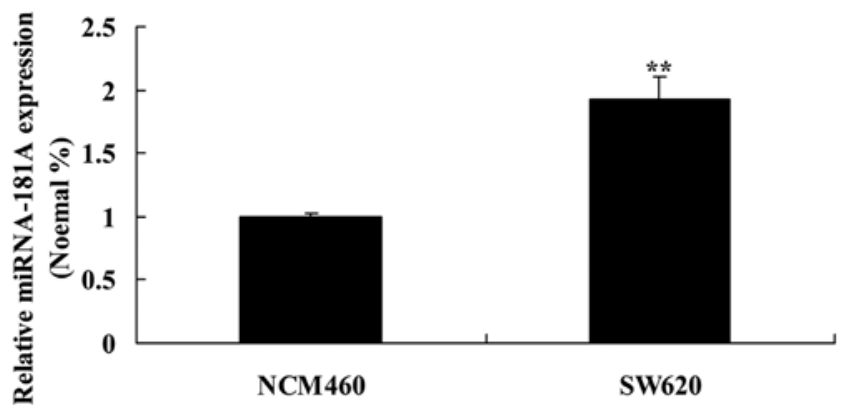

Figure 4. The expression of miR-181A in NCM460 and SW620 cells. ${ }^{* * *} \mathrm{P}<0.01$ compared with normal group.
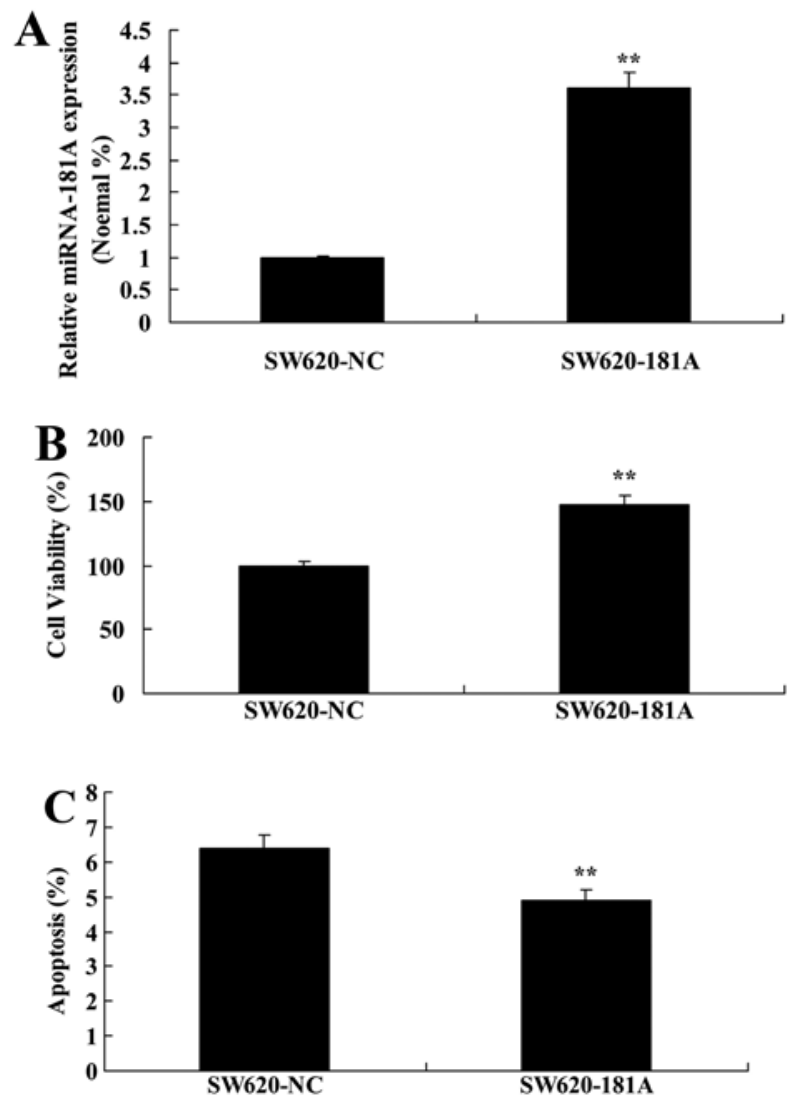

Figure 5. miR-181A induces cell growth and inhibits cell apoptosis of SW620 cells. miR-181A expression of SW620 cells (A), miR-181A induces cell growth (B) and inhibits (C) cell apoptosis of SW620 cells. ${ }^{* *} \mathrm{P}<0.01$ compared with the normal control group.

The expression of miR-181A in NCM460 and SW620 cells. To explore the functional role of miR-181A in driving late liver metastases, we examined the miR-181A expression using qRT-PCR analyses in the cell lines NCM460 and SW620. The miR-181A expression in the colon cancer cells NCM460 was lower than that of the colon cancer SW620 cells (Fig. 4).

miR-181A induces cell growth and inhibits apoptosis of $S W 620$ cells. We examined how the upregulation of miR-181A could affect cell growth and cell apoptosis of SW620 cells, and found that miR-181A plasmid increased the expression of miR-181A in SW620 cells (Fig. 5A). Moreover, upregulation of 


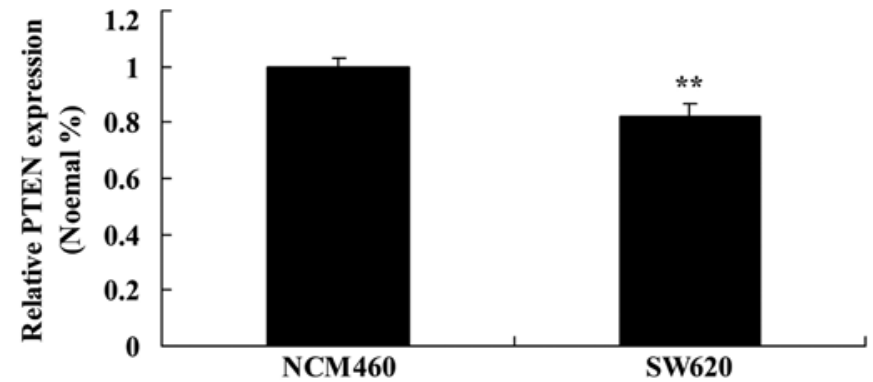

Figure 6. The expression of PTEN in NCM460 and SW620 cells. ${ }^{* * *} \mathrm{P}<0.01$ compared with normal group.

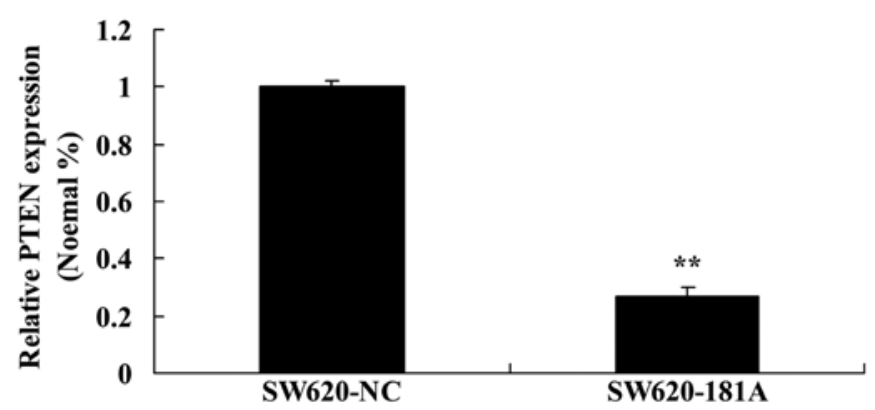

Figure 7. miR-181A inhibits the PTEN expression of SW620 cells.

A

p-AKT

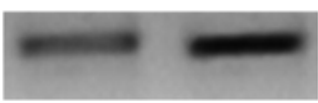

AKT

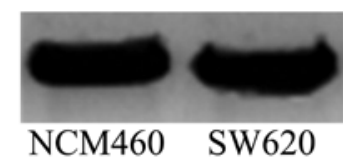

B

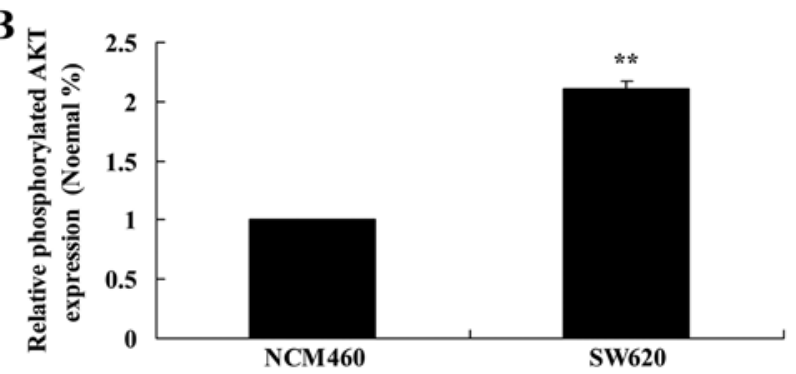

Figure 8. The expression of AKT in NCM460 and SW620 cells. ${ }^{* *} \mathrm{P}<0.01$ compared with normal group.

miR-181A enhanced cell viability and inhibited cell apoptosis of SW620 cells (Fig. 5B and C).

The expression of PTEN in NCM460 and SW620 cells. To study the role of PTEN in CRC patients with late liver metastases, we evaluated the PTEN expression using qRT-PCR in NCM460 and SW620 cells. Consistent with in vitro results, the expression of PTEN in NCM460 cells was lower than that of PTEN expression in SW620 cells (Fig. 6).

miR-181A inhibits the PTEN expression of SW620 cells. Notably, miR-181A plasmid increased the expression of PTEN

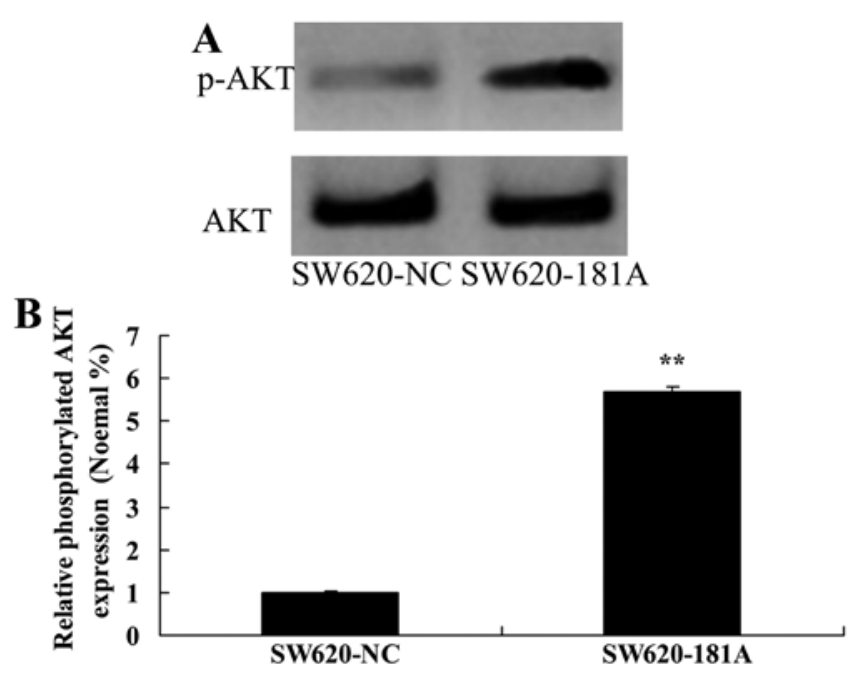

Figure 9. miR-181A induces the AKT expression of SW620 cells. miR-181A induces the p-AKT and AKT protein expression of SW620 cells using western blot analysis (A), statistical analysis of p-AKT and AKT protein expression (B). ${ }^{* *} \mathrm{P}<0.01$ compared with the control group.

in SW620 cells (Fig. 7). These results indicate that PTEN may participate in the effect of miR-181A on SW620 cells. However, the mechanism of such an interaction is unknown.

The expression of AKT in NCM460 and SW620 cells. To explore the AKT signaling pathway, western blotting was performed in NCM460 and SW620 cells. Antibodies were used against AKT and phosphorylated-AKT to examine protein expression. The expression of phosphorylated AKT in NCM460 cells was lower than that of SW620 cells (Fig. 8).

miR-181A induces AKT expression in SW620 cells. Notably, miR-181A plasmid suppressed the expression of phosphorylated AKT protein in SW620 cells (Fig. 9). This suggests that miR-181 affects the AKT pathway in human cancer SW620 cells.

Downregulation of miR-181A inhibits cell growth of SW620 cells via modulation of the PTEN/AKT signal pathway. Anti-miR-181A plasmid was able to reduce the expression of miR-181A in SW620 cells (Fig. 10A). Anti-miR-181A plasmid also inhibited cell viability of SW620 cells (Fig. 10B). The expression of PTEN was activated and the expression of phospho-AKT was suppressed by anti-miR-181A plasmid (Fig. 10C-E).

\section{Discussion}

Colorectal cancer is one of the most common malignant types of cancer, worldwide, with mortality rates of CRC ranking third. According to data in 2008, there were nearly 1 million new cases of CRC and 500,000 deaths as a result of CRC, worldwide (12). Despite the rapid advance of endoscopic techniques, the improvement in material living standards, and changes in dietary patterns, the morbidity of CRC in China is increasing (13). In the 1990s, the morbidity of CRC in urban and rural areas in China increased by 32 . and $8.5 \%$, respectively, compared with that in 1970s. In China, CRC 

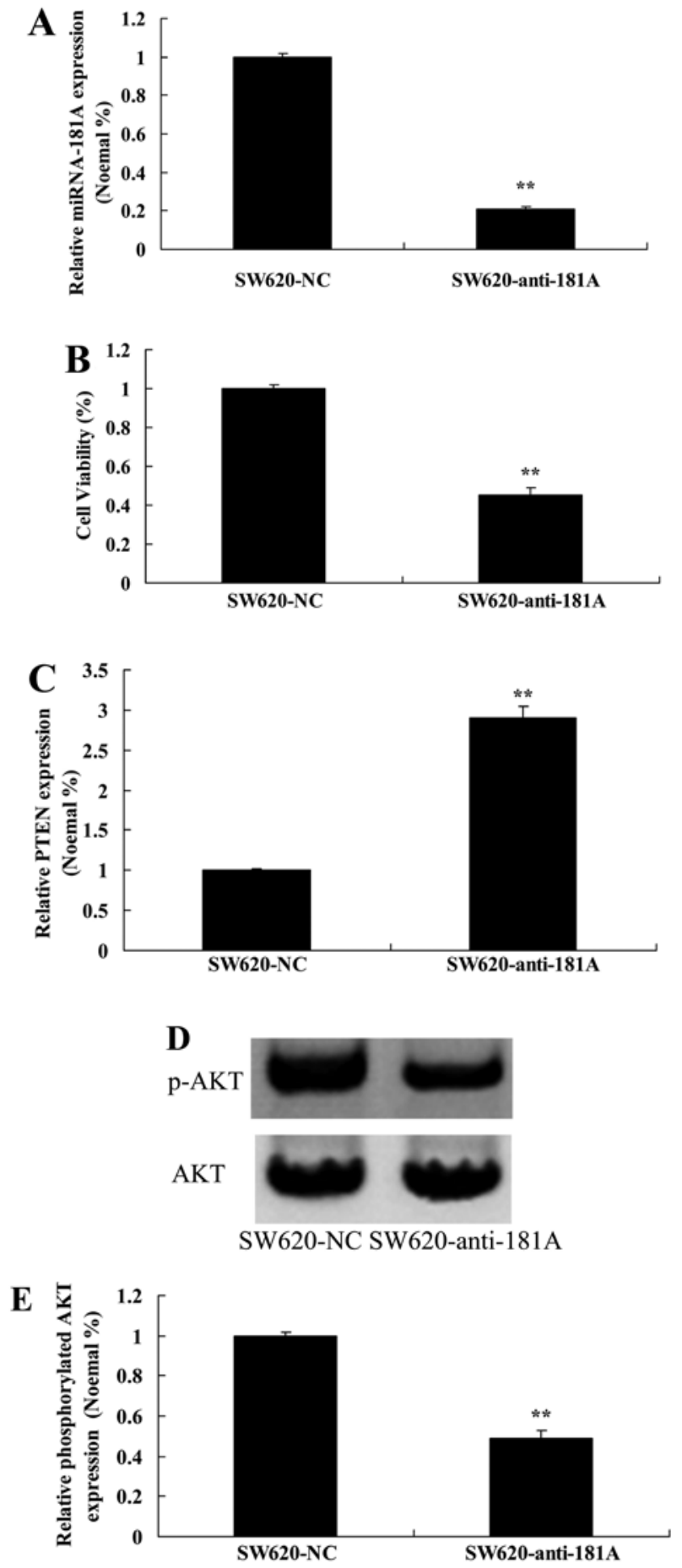

Figure 10. Downregulation of miR-181A inhibits cell growth of SW620 cells through mediating the PTEN/AKT signal pathway. miR-181A expression of SW620 cells (A), miR-181A induces cell growth (B) and PTEN expression (C), p-AKT and AKT protein expression of SW620 cells using western blot analysis (D), statistical analysis of p-AKT and AKT protein expression (E) of SW620 cells. ${ }^{* *} \mathrm{P}<0.01$ compared with normal group.

has become the most common malignant cancer and a major health issue (13). We found that the expression of miR-181A in CRC patients with late liver metastases was higher than that of the normal group and that the expression of miR-181A was lower in all the pathological groups (TNM I-TNM IV).

With a length of 19-24 nucleotides, miRNA is a non-coding small RNA, which can exert a regulatory effect by combing the untranslated region (3'-UTR) (14). Nearly 1,500 miRNA sequences have been discovered, to date and $>60 \%$ of genes reported in the human genome are miRNA target genes (15). It has been shown that miRNA can regulate the expression of numerous genes and that miRNAs play an important role in normal cell processes, such as cell growth and development, proliferation and apoptosis (14). Increasing evidence reveals that miRNAs play a crucial role in many types of cancer (16), and indeed many tumour cells have altered expression of miRNAs (17).

In the present study, we found that there was an increase of OS in lower expression miR-181A of CRC patients with late liver metastases compared to CRC patients with higher miR-181A levels with late liver metastases. Specifically, upregulation of miR-181A increased the cell viability and inhibited apoptosis of SW620 cells.

It has been reported that miR-181A has many different target genes. PTEN is a tumor suppressor gene and has dual phosphatase activity on lipid and proteins to drive processes essential in cell processes, such as cell growth, proliferation, survival, apoptosis, migration and invasion, local adhesion and angiogenesis (18). Mutations that drive upregulation or deletion of PTEN can activate the PI3K/Akt signaling pathway which can trigger occurrence, development, invasion and metastasis of tumors (19). Studies showing that miR-181 A can target the regulation of PTEN and promote occurrence, development and metastasis of tumors are common, while studies examining the related regulatory mechanisms in CRC are rare $(18,20)$. In the present study, we found that the PTEN expression of the normal control patients was higher than that of CRC patients with late liver metastases. Moreover, PTEN expression of TNM I CRC patients was markedly enhanced compared to other pathological groups. When we upregulated miR-181A using an expression plasmid, the expression of PTEN in SW620 cells increased.

Studies have suggested that the PTEN/PI3K/Akt signal pathway is involved in numerous cell processes, such as growth, proliferation, survival, apoptosis, metabolism, angiogenesis, invasion and metastasis, anticancer drug resistance and tumor immune escape (21). However, reports examining miR-181A and its regulation of the PTEN/PI3K/Akt signal pathway are rare. In this study we examined the expression of related expression of miR-181A, PTEN/PI3K/Akt signaling pathways in CRC tissues to understand its clinical significance. We would like to extend this study to verify that miR-21 is a direct target gene and to examine regulatory mechanisms of the PTEN/PI3K/Akt signal pathway by miR-181A. Ultimately, we hope to understand how miR-181A might regulate biological behavior of CRC, such as proliferation, invasion, metastasis and cell cycle progression, as this might help provide clues for new therapeutic targets.

Specifically we found that upregulation of miR-181A induced AKT expression in SW620 cells. Downregulation of miR-181A could suppress cell growth of SW620 cells, and mediated the PTEN/AKT signaling pathway.

In conclusion, we report that the expression of miR-181A in CRC patients with late liver metastases was higher than that of the normal group. Downregulation of miR-181A was able to suppress growth of SW620 cells by mediating the 
PTEN/AKT signal pathway. Our results support the idea that miR-181A affects incidence and survival in late liver metastases of colorectal cancer through the PTEN/AKT signaling pathway.

\section{Acknowledgements}

This study was funded by the Science and Technology Development Plan Project of Shandong Province, China (no. 2013GSF11834), the Shandong Provincial Natural Science Foundation, China (no. ZR2009CM138) and the Medicine and Health Care in Shandong Province Science and Technology Development Plan Project (no. 2011HW06).

\section{References}

1. Li Y, Zeng M, Chen W, Liu C, Wang F, Han X, Zuo Z and Peng S Dexmedetomidine reduces isoflurane-induced neuroapoptosis partly by preserving PI3K/Akt pathway in the hippocampus of neonatal rats. PLoS One 9: e93639, 2014.

2. Van Schaeybroeck S, Kalimutho M, Dunne PD, Carson R, Allen W, Jithesh PV, Redmond KL, Sasazuki T, Shirasawa S, Blayney J, et al: ADAM17-dependent c-MET-STAT3 signaling mediates resistance to MEK inhibitors in KRAS mutant colorectal cancer. Cell Rep 7: 1940-1955, 2014.

3. Maekawa K, Baba T, Otomo S, Morishita S and Tamura N: Low pre-existing gray matter volume in the medial temporal lobe and white matter lesions are associated with postoperative cognitive dysfunction after cardiac surgery. PLoS One 9: e87375, 2014

4. Cai Y, Hu H, Liu P, Feng G, Dong W, Yu B, Zhu Y, Song J and Zhao M: Association between the apolipoprotein E4 and postoperative cognitive dysfunction in elderly patients undergoing intravenous anesthesia and inhalation anesthesia. Anesthesiology 116: 84-93, 2012

5. Zhang Q, Li SZ, Feng CS, Qu XD, Wang H, Zhang XN, Liu Y, Wang Y, Wu AS and Yue Y: Serum proteomics of early postoperative cognitive dysfunction in elderly patients. Chin Med J (Engl) 125: 2455-2461, 2012.

6. Lin K and Abraham KM: Targets of p56(lck) activity in immature thymoblasts: Stimulation of the Ras/Raf/MAPK pathway. Int Immunol 9: 291-306, 1997.

7. Kleist B, Kempa M, Novy M, Oberkanins C, Xu L, Li G, Loland C and Poetsch M: Comparison of neuroendocrine differentiation and KRAS/NRAS/BRAF/PIK3CA/TP53 mutation status in primary and metastatic colorectal cancer. Int J Clin Exp Pathol 7: 5927-5939, 2014.

8. Sinha R, Larkin J, Gore M and Fearfield L: Cutaneous toxicities associated with vemurafenib therapy in 107 patients with BRAF V600E mutation-positive metastatic melanoma, including recognition and management of rare presentations. Br J Dermatol 173 : 1024-1031, 2015.
9. Okamoto K, Okamoto I, Hatashita E, Kuwata K, Yamaguchi H, Kita A, Yamanaka K, Ono M and Nakagawa K: Overcoming erlotinib resistance in EGFR mutation-positive non-small cell lung cancer cells by targeting survivin. Mol Cancer Ther 11: 204-213, 2012.

10. Cercek A, Shia J, Gollub M, Chou JF, Capanu M, Raasch P, ReidyLagunes D, Proia DA, Vakiani E, Solit DB, et al: Ganetespib, a novel Hsp90 inhibitor in patients with KRAS mutated and wild type, refractory metastatic colorectal cancer. Clin Colorectal Cancer 13: 207-212, 2014.

11. Giannoni E, Fiaschi T, Ramponi G and Chiarugi P: Redox regulation of anoikis resistance of metastatic prostate cancer cells: Key role for Src and EGFR-mediated pro-survival signals. Oncogene 28: 2074-2086, 2009.

12. Whelan KA, Schwab LP, Karakashev SV, Franchetti L, Johannes GJ, Seagroves TN and Reginato MJ: The oncogene HER2/neu (ERBB2) requires the hypoxia-inducible factor HIF-1 for mammary tumor growth and anoikis resistance. J Biol Chem 288: 15865-15877, 2013

13. Oh HY, Lee EJ, Yoon S, Chung BH, Cho KS and Hong SJ: Cholesterol level of lipid raft microdomains regulates apoptotic cell death in prostate cancer cells through EGFR-mediated Akt and ERK signal transduction. Prostate 67: 1061-1069, 2007.

14. Roomi MW, Kalinovsky T, Rath M and Niedzwiecki A: In vitro modulation of MMP-2 and MMP-9 in pediatric human sarcoma cell lines by cytokines, inducers and inhibitors. Int J Oncol 44: 27-34, 2014.

15. Lawless N, Vegh P, O'Farrelly C and Lynn DJ: The role of microRNAs in bovine infection and immunity. Front Immunol 5: 611,2014

16. Cortés-Sempere $M$ and Ibáñez de Cáceres I: microRNAs as novel epigenetic biomarkers for human cancer. Clin Transl Oncol 13: 357-362, 2011.

17. Rotomskis A, Margevičiūtè R, Germanavičius A, Kaubrys G, Budrys V and Bagdonas A: Differential diagnosis of depression and Alzheimer's disease with the Addenbrooke's Cognitive Examination-Revised (ACE-R). BMC Neurol 15: 57, 2015.

18. Yen SS: Proteasome degradation of brain cytosolic tau in Alzheimer's disease. Int J Clin Exp Pathol 4: 385-402, 2011.

19. Zheng J, Wu C, Xu Z, Xia P, Dong P, Chen B and Yu F: Hepatic stellate cell is activated by microRNA-181b via PTEN/Akt pathway. Mol Cell Biochem 398: 1-9, 2015.

20. Jun Z, Li Z, Fang W, Fengzhen Y, Puyuan W, Wenwen L, Zhi S and Bondy SC: Melatonin decreases levels of S100 $\beta$ and NFKB, increases levels of synaptophysin in a rat model of Alzheimer's disease. Curr Aging Sci 6: 142-149, 2013.

21. Granic I, Dolga AM, Nijholt IM, van Dijk G and Eisel UL: Inflammation and NF-kappaB in Alzheimer's disease and diabetes. J Alzheimers Dis 16: 809-821, 2009. 